\title{
Plataformas de Serviços de Bibliotecas: a evolução dos sistemas para gerenciamento de bibliotecas
}

\author{
Fabiana John Tonding ${ }^{I}$ \\ Samile Andréa de Souza Vanz ${ }^{I I}$ \\ I Universidade Federal do Rio Grande do Sul, Programa \\ de Pós-Graduação e Comunicação e Informação, Porto Alegre, RS, Brasil. \\ Mestre em Comunicação e Informação do Programa \\ de Pós-Graduação em Comunicação e Informação \\ da Universidade Federal do Rio Grande do Sul.
}

\begin{abstract}
II Universidade Federal do Rio Grande do Sul, Programa de Pós-Graduação em Comunicação e Informação, Departamento de Ciências da Informação, Porto Alegre, RS, Brasil.

Professora associada do Departamento de Ciências da Informação, do Programa de Pós-graduação em Comunicação e Informação

do Programa de Pós-graduação em Ciência da Informação da Universidade

Federal do Rio Grande do Sul.
\end{abstract}

http://dx.doi.org/10.1590/1981-5344/3302

O artigo apresenta uma revisão de literatura sobre as plataformas de serviços de bibliotecas (library services platforms - LSP). Discorre sobre o histórico e a evolução da informatização de bibliotecas e também descreve algumas tecnologias, ferramentas e recursos utilizados até o surgimento das plataformas de serviços de bibliotecas. São apresentadas as características das LSPS: possibilidade para gerenciar coleções de itens físicos e eletrônicas; suporte a vários processos de aquisição; oferta de um ambiente híbrido de gerenciamento de metadados, incluindo, no mínimo, os padrões MARC e Dublin Core; inclusão de serviço de descoberta integrado ou uso de uma interface de descoberta adquirida; operação através do modelo Service-as-a-Software e de estrutura multi-tenant; e, acesso via interface web. 0 artigo conclui que, apesar de as bibliotecas brasileiras ainda não utilizarem as plataformas de serviços de bibliotecas, é fundamental discutir o tema tendo em vista sua relevância e atualidade. 
Palavras-chave: Plataformas de serviços de bibliotecas. Sistemas para gerenciamento de bibliotecas. Informatização de bibliotecas. Serviços em nuvem para bibliotecas.

\section{Library Services Platforms: the evolution of the library management systems}

The article presents a literature review about library services platforms (LSP). The history and evolution of libraries automation is addressed, and it also covers some technologies, tools and resources that have been used by libraries until the emergence of the library services platforms. The LSP characteristics are explored: they support multiple procurement processes, spanning from physical items to electronic resources; offer hybrid metadata tools, including at least MARC and Dublin Core schemas appropriate to each material; include an integrated discovery service or support a separately acquired one; are provided as subscription, based on Software-as-a-Service model and deployed through multitenant platforms; staff interfaces are offered through web browsers. Although Brazilian libraries still do not use the library services platforms, it concludes that it is essential to discuss the theme because of its relevance and contemporaneity.

Keywords: Library services platforms. Library management systems. Library automation. Cloud services for libraries.

Recebido em 05.10.2017 Aceito em 22.05.2018

\section{Introdução}

O comportamento do usuário das bibliotecas universitárias, no que diz respeito à geração, à busca, ao acesso e à comunicação da informação científica e tecnológica, foi alterado pela web (OCLC, 2011a). O domínio da internet, a variedade de suportes e de fontes de informação e o ensino à distância foram fenômenos que provocaram mudanças nas instituições de ensino e a migração do sujeito usuário de biblioteca para usuário de informação. Neste cenário, há novas oportunidades e novos desafios que exigem das bibliotecas universitárias um posicionamento centrado em seu 
papel junto à sua comunidade, condizente com a realidade educacional atual.

Para recuperar a centralidade como instituição de apoio educacional, dedicada à aprendizagem e ao conhecimento, a biblioteca deve rever algumas práticas e muitos procedimentos de seu cotidiano. Precisa se reestruturar e se renovar. Chien e Tsaur (2007) esclarecem que as organizações, atualmente, buscam formas de melhorar o desempenho e sustentar vantagens competitivas por meio do desenvolvimento efetivo dos recursos e dos processos de negócios. E afirmam que a chave da competitividade reside em dispor de sólida infraestrutura de sistema de informação, alinhada com os processos centrais realizados para entrega de serviços e produtos aos clientes dentro do melhor tempo (CHIEN; TSAUR, 2007). A adoção de sistemas de gerenciamento contemporâneos e inovadores é, portanto, um dos elementos que permite a concretização da mudança necessária às bibliotecas.

Com este enfoque, desde o início desta década, observa-se, principalmente, entre bibliotecas de instituições de ensino superior e de pesquisa, a adoção de plataformas de serviços de bibliotecas (em inglês library services platforms - LSP). Esta nova geração de sistemas, desenvolvidos com tecnologias recentes e providos através de serviços em nuvem, contemplam o gerenciamento unificado dos mais diversos recursos de informação hoje existentes nas bibliotecas - sejam eles impressos, eletrônicos ou digitais. As soluções de LSP compreendem funcionalidades voltadas para a operacionalização das atividades internas da biblioteca e se integram aos serviços de descoberta para as funções de busca e de acesso às informações pelos usuários finais.

As plataformas de serviços de bibliotecas são fornecidas através do modelo Software-as-a-Service (SaaS) - no qual o software não é mais comprado e instalado, mas usado por meio de uma assinatura. O modelo SaaS é considerado uma tecnologia disruptiva que gera novos processos, novas formas de fazer, criando um novo valor. Um dos aspectos apontados pela literatura é o quanto as plataformas de bibliotecas desoneram a equipe da biblioteca e da área de tecnologia da informação para desenvolverem atividades relevantes e inovadoras (DULA et al., 2012; GOLDNER, 2012; GRANT, 2012a). O ganho de produtividade se dá através da otimização de fluxos de trabalho que o sistema contempla e também porque, dada sua implementação em nuvem, as equipes de TI ficam eximidas de atividades rotineiras de administração dos recursos de hardware e software. Com esta força de trabalho adicional, as bibliotecas podem centrar esforços em atividades que agregam valor e, assim, recuperar seu papel fundamental no processo de ensino, aprendizagem e pesquisa.

Embora nova, há indícios de que esta geração de sistemas de gerenciamento está se consolidando. Nos países estrangeiros, o uso das plataformas de serviços de bibliotecas vem aumentando gradualmente 
desde 2012 (LIBRARY..., 2017) ${ }^{1}$. No Brasil, por sua vez, não há bibliotecas utilizando as LSPs e é incipiente o interesse por estas soluções. É natural, no entanto, que as bibliotecas brasileiras passem a analisar esta tendência e avaliar a adoção dos novos sistemas com o amadurecimento da discussão sobre o tema.

Este artigo tem como objetivo explorar o fenômeno das plataformas de serviços de bibliotecas, alicerçando a temática das LSPs na literatura acadêmica nacional, dada a inexistência de pesquisas anteriores sobre o tema. Os procedimentos metodológicos se fundamentam em uma revisão de literatura realizada em abril de 2017 no Portal de Periódicos da Coordenação de Aperfeiçoamento de Pessoal de Nível Superior (CAPES) e no Google Acadêmico, utilizando-se as expressões de busca: plataforma de serviços de bibliotecas, library services platform, LSP, sistema de nova geração, next generation system, webscale management system, sistema de automação de biblioteca, library automation system, sistema AND biblioteca $A N D$ nuvem, system AND library AND cloud e suas variações em relação a grafias e plurais. Nos documentos localizados no Portal de Periódicos da CAPES fez-se uma exploração das referências citadas para expandir a relação de trabalhos sobre o tema. Foram identificados 49 artigos relacionados às plataformas de serviços de bibliotecas, em sua maioria textos estrangeiros com concepções descritivas, acerca do surgimento dos novos sistemas, relatos de experiências do processo de seleção e adoção e, principalmente, sobre suas inovadoras características tecnológicas e funcionais.

A revisão de literatura realizada permitiu desenvolver, na primeira seção deste artigo, o histórico da informatização das bibliotecas e sua evolução até o surgimento dos sistemas integrados de bibliotecas (ILS, do inglês Integrated Library System), descrevendo também algumas tecnologias, ferramentas e recursos: os catálogos on-line (OPAC), as publicações eletrônicas, o OpenURL, as bibliotecas e os repositórios digitais, as soluções ERMS, os sistemas de busca federada e os serviços de descoberta. A seção seguinte discorre sobre as novas plataformas de serviços de bibliotecas e suas características. O artigo encerra com a seção de considerações finais e as principais referências pesquisadas na revisão de literatura.

\section{Evolução dos sistemas para gerenciamento de recursos de bibliotecas}

A explosão informacional e o aumento da oferta e da busca por publicações eletrônicas mudaram os processos das bibliotecas e o uso dos recursos informacionais por seus usuários finais. Os acervos, compostos por materiais impressos e eletrônicos, envolvem novas formas de aquisição, de registro, de disponibilização e acesso, de controle e de utilização. A crescente predileção por conteúdos eletrônicos e decrescente

Dados de migraçã disponívei no site do Library Technology Guides. Disponível em: <https://librarytechnology.org/products/migration.pl>. Acesso em: 6 maio 2017. 
por materiais impressos demanda ferramentas que quebrem os modelos tradicionais de gerenciamento de bibliotecas e atendam a uma visão mais ampla de suas coleções (BREEDING, 2011).

A evolução dos sistemas para gerenciamento de bibliotecas tem sua trajetória pautada pelo surgimento e disponibilização de novas tecnologias, que permitem que os sistemas sejam modernizados, visando atender as necessidades técnicas e informacionais contemporâneas das bibliotecas e de seus usuários internos e finais. Rowley (2002) estabeleceu um enquadramento dos sistemas de informatização de bibliotecas em quatro gerações, que permeiam avanços tecnológicos, conforme segue:

a) a primeira geração se caracterizava por sistemas com foco na catalogação e na circulação, na forma de módulos independentes, sem integração entre estes módulos. Desenvolvidos com linguagem, sistema operacional e sistema de gerenciamento de banco de dados proprietários, os sistemas rodavam em plataformas de equipamentos específicas. Alguns foram desenvolvidos para serem comercializados juntamente com os equipamentos. O uso dos sistemas se dava através de comandos, sem nenhuma facilidade de interface;

b) na segunda geração, os fabricantes passaram a preocuparse com a interligação de módulos, mas as interfaces ainda eram rudimentares e a utilização dos sistemas dependia de menus. Apareceram as primeiras possibilidades de exportação e importação de registros, porém restritas a sistemas específicos. As estruturas das bases de dados ainda eram proprietárias;

c) a terceira geração de sistemas para gerenciamento de bibliotecas disponibilizou relatórios padronizados e a opção para a biblioteca definir aqueles de seu interesse, conforme as necessidades dos gestores. A utilização de cores, janelas, ícones e menus, tornou a interface gráfica e mais amigável. Com o aparecimento de sistemas baseados em UNIX $^{2}$ e DOS $^{3}$, os sistemas passaram a rodar em uma variedade de plataformas, tornando-se mais portáteis e não restritos a um fornecedor. Gradativamente passaram a adotar padrões de comunicação que garantiam a interconexão com outros sistemas;

d) na quarta geração, a importação e exportação de registros passou a ser totalmente integrada e facilitada, a arquitetura

\footnotetext{
${ }^{2}$ Unix é um sistema operacional criado originalmente por Ken Thompson, Dennis Ritchie, Douglas McIlroy e Peter Weiner, que trabalhavam nos Laboratórios Bell (Bell Labs) da AT\&T. Disponível em: <https://pt.wikipedia.org/wiki/Unix>. Acesso em: 24 abr. 2017.

${ }^{3}$ DOS é a sigla para Disk Operating System ou sistema operacional em disco, acrônimo para diversos sistemas operacionais que dominaram o mercado de compatíveis IBM PC. Disponível em: <https://pt.wikipedia.org/wiki/DOS>. Acesso em: 24 abr. 2017.
} 
cliente-servidor permitiu o acesso a outros servidores da internet e os usuários finais podiam consultar os catálogos a partir de múltiplas mídias. Observa-se a utilização de linguagens orientadas a objetos e a disponibilidade de sistemas para o ambiente Windows. Surgem os catálogos on-line (em inglês, Online Public Access Catalog - OPAC, sigla utilizada neste estudo), com grande avanço na qualidade da interface. Há possibilidade de adequação das características gráficas da interface, de oferecer diversas opções de buscas, de exibir os registros em diferentes formatos e de dar acesso a diferentes subconjuntos de registros da base dados. A flexibilidade de configurações do OPAC revelou uma mudança no foco do desenvolvimento, voltado para os usuários finais, contrariamente ao observado nos sistemas primitivos, cujos projetos focavam nas necessidades da equipe técnica das bibliotecas. Com a disseminação da internet, o acesso aos OPACs, primeiramente oferecidos localmente nos espaços das bibliotecas, passou a ser remotamente.

Os atributos da quarta geração são aplicáveis aos sistemas integrados de bibliotecas. Conforme Tyagi e Senthil (2015), os ILSs são softwares baseados em arquitetura cliente-servidor, acessíveis aos usuários através da rede local (LAN), e operam de forma interligada e coordenada para automatização das "[...] atividades relacionadas com aquisição, circulação, gerenciamento de usuários finais, gerenciamento de livros, ajudas, interações entre bibliotecas, indexação, etc [...]" (TYAGI; SENTHIL, 2015, p. 409, tradução nossa). A adoção dos sistemas integrados de bibliotecas tornou-se predominante nos anos 80 e 90 (WANG; DAWES, 2012) e esta geração é ainda prevalente, tendo em vista que grande parte dos sistemas atuais de automação de bibliotecas consagrados são da era cliente/servidor (BREEDING, 2011).

Cabe lembrar que no Brasil as primeiras iniciativas de automação de bibliotecas surgiram na década de 80 , com o desenvolvimento de sistemas pelas próprias instituições - entre as quais destacaram-se as universidades - promovendo um significativo avanço às bibliotecas acadêmicas (VIANA, 2016). No início dos anos 90, com a abertura do mercado de informática, foi possível a aquisição de equipamentos e de sistemas estrangeiros mais modernos. No cenário atual brasileiro, entre os softwares para gerenciamento de bibliotecas com maior difusão entre as universidades destacam-se o Aleph (Ex Libris/ProQuest), o Pergamum (Pontifícia Universidade Católica do Paraná) e o SophiA Biblioteca (Prima) que, dado às suas estruturas e características, podem ser considerados como sistemas de quarta geração.

Um dos destaques dos sistemas de quarta geração são os OPACs, que permitem a busca dos recursos das bibliotecas nos sistemas de tipo ILS. É importante ressaltar que as funcionalidades dos ILSs, incluindo os OPACs, foram concebidas e desenvolvidas com foco apenas em recursos 
impressos, tipo documental predominante à época do surgimento destas soluções. Li (2014) diz que à medida que os conteúdos eletrônicos têm maior peso nas coleções das bibliotecas, a fraqueza dos ILSs e dos OPACs torna-se evidente. Pavão (2014) comenta que os OPACs são amplamente utilizados pela maioria das bibliotecas como ferramenta de apoio à pesquisa, mas ressalta que, por serem originados em uma fase préinternet, apresentam limitações quando comparados com outras ferramentas de pesquisa na web que ganharam proeminência e popularidade, como por exemplo, a notória ferramenta de busca do Google.

A internet é um divisor da linha do tempo das bibliotecas. São muitas as mudanças advindas nos processos das bibliotecas e na cadeia produtiva em que elas estão inseridas. Observa-se efeitos no que tange a fornecedores de sistemas e de publicações, formas de geração, organização e registro da documentação, meios de acesso à informação e a documentos, comportamento de usuários finais e relacionamento institucional e social. Em decorrência da internet, constata-se o surgimento de uma série de recursos, de dispositivos, de sistemas e de ferramentas para apoiar a biblioteca e seus usuários finais e internos nesta nova era.

Um destes recursos é o mecanismo OpenURL, que expande os links no ambiente da informação científica, enriquecendo a pesquisa e a exploração dos recursos informacionais com serviços adicionais e criativos. Esta tecnologia é definida pela norma OpenURL da National Information Standards Organization (NISO) e foi inicialmente desenvolvida por Van de Sompel e Oren Beit-Arie (APPS; MACINTYRE, 2005). Além de ampliar os serviços oferecidos sobre um recurso eletrônico, o OpenURL oferece links sensíveis ao contexto do usuário final. Um exemplo é a exibição de links que levam à fonte eletrônica a qual a biblioteca possui acesso, expondo e aumentando a visibilidade dos recursos disponíveis aos pesquisadores, sem criar frustração de exibir materiais aos quais não terão acesso (VAN DE SOMPEL; BEIT-ARIE, 2001). Amplamente utilizado em soluções chamadas resolvedores de links (em inglês, link resolvers), o mecanismo de OpenURL é essencial para as bibliotecas utilizarem e explorarem de maneira inteligente suas coleções eletrônicas.

Além das coleções eletrônicas, compostas de livros, periódicos e bases de dados, fornecidas mediante licenciamento ou de acesso livre, as bibliotecas também passaram a lidar com os acervos em formato digital. As bibliotecas digitais, em geral, abrigam acervos que as instituições detêm de forma característica ou exclusiva - fotografias, monografias e periódicos antigos, coleções especiais, materiais raros ou únicos, que muitas vezes são parte das coleções das bibliotecas, e que podem ser interessantes para os pesquisadores se ficarem à disposição para consulta e acesso via web (ABADAL; ANGLADA, 2017). Para a construção e operação de bibliotecas digitais existem alternativas de sistemas comerciais e de código aberto (em inglês, open source) e uma série de 
requisitos tecnológicos a serem seguidos, como de interoperabilidade e de preservação, entre outros.

Tecnologicamente semelhantes às bibliotecas digitais, porém com outro propósito e abrangência, surgiram os repositórios digitais, que a partir de meados da década de 2000, difundiram-se como uma nova forma de acesso à ciência, pois tornam a produção intelectual livre e abertamente disponível. Desta forma, estas iniciativas "[...] vêm construindo as condições necessárias para permitir o acesso livre à produção científica de forma legítima, alterando não somente o processo de aquisição de informação científica, mas também a sua produção, disseminação e uso" (WEITZEL, 2006, p. 52). Do ponto de vista operacional, as bibliotecas universitárias e de instituições de pesquisa podem estar envolvidas com as questões e processos atinentes às bibliotecas e aos repositórios digitais e, inclusive, no caso destes últimos, "[...] avançando em novas áreas como gerenciamento de dados de pesquisa e mesmo de publicação eletrônica" (CHAD, 2016, p. 3, tradução nossa).

Outra ferramenta complementar às atividades das bibliotecas são os sistemas para gerenciamento de recursos eletrônicos, tradução de Electronic Resources Management System (ERMS). Os ERMS oferecem funcionalidades para seleção, aquisição, licenciamento, acesso, manutenção, acompanhamento e avaliação de uso, renovação ou cancelamento de fontes eletrônicas. As fontes podem ser de livre acesso ou licenciadas e incluem periódicos, livros, bases de dados, anais de eventos e outros materiais e recursos acadêmicos de natureza on-line. Conforme Noe (2013), o controle de alguns ou mesmo milhares de títulos, e os respectivos dados de seus editores ou fornecedores, é possível de ser feito utilizando uma planilha ou um arquivo qualquer, sem necessidade de um ERMS. Mas, no caso de pacotes de títulos eletrônicos, surgem questões complexas de responder que dependem de dados melhor estruturados por um sistema próprio para gerenciamento de recursos eletrônicos.

Segundo Anderson (2014), um mesmo periódico pode estar disponível em diversas plataformas, providas por diferentes fornecedores, acessíveis em múltiplos endereços eletrônicos (URL ${ }^{4}$ ) e sob diferentes condições de licenciamento. Os ERMS pretendem solucionar a representação dos dados necessários para o gerenciamento de recursos eletrônicos, através do desenvolvimento de uma base de dados de conhecimento (KB, do inglês, Knowledge Base). A base de conhecimento contém informações sobre estes recursos eletrônicos, incluindo os metadados dos títulos disponíveis para compra ou assinatura, plataformas, preços, termos de licenciamento e condições de uso legal do conteúdo. Coletar estes dados acerca dos recursos eletrônicos dos mais diversos editores, publicadores e agregadores e manter a base de conhecimento atualizada é uma tarefa árdua - o que Breeding (2012b)

4 "[...] URL - Uniform Resource Locator - é a porta de entrada para os conteúdos que estão disponíveis na Web. Ela define, como seu próprio nome diz, a localização do recurso" (SAYÃO, 2007, p. 67). 
confirma, destacando que, para criar e manter estas bases de conhecimento de conteúdo eletrônico, são necessários significativos recursos. Esta complexidade de informações, claramente contidas nas bases de conhecimento dos ERMS, é difícil de ser controlada e rastreada em um sistema tradicional de gerenciamento de bibliotecas, do tipo ILS (ANDERSON, 2014). Por este motivo, alguns fornecedores de ILS começaram a desenvolver módulos de ERMS para serem integrados aos sistemas.

Visando facilitar a busca em múltiplas fontes de conteúdo simultaneamente - como por exemplo, nos catálogos das bibliotecas, nas revistas eletrônicas, nas bases de dados, nos repositórios digitais, entre outros recursos - surgiram na década passada os metabuscadores ou ferramentas de busca federada, com aplicação do protocolo Z39.50. A tecnologia deu agilidade à pesquisa, permitindo explorar múltiplos recursos a partir de uma única busca, mas "[...] por outro lado o tempo de resposta para apresentação dos resultados não tem sido satisfatório, visto que os metabuscadores utilizam mecanismos de busca de cada uma das fontes pesquisadas" (PAVÃO, 2014, f. 16).

A insatisfação com a falta de uma ferramenta de busca unificada, o tempo necessário para pesquisa em diversas fontes e a necessidade de conhecer os detalhes de acesso e as interfaces de diferentes provedores motivaram o surgimento dos sistemas de descoberta e entrega:

O princípio básico dos serviços de descoberta é fornecer um índice único de metadados [...] previamente coletados que permita uma busca unificada substituindo a busca federada, ou seja, a busca em cada uma das bases provedoras de conteúdos, utilizada até então. Hospedados local ou remotamente, disponibilizam uma variedade de informações que podem incluir todo tipo de conteúdo licenciado, conteúdo local e o próprio catálogo da biblioteca, tudo combinado num índice único. (PAVÃO, 2014, f. 16).

Atualmente, com a adoção dos sistemas de descoberta em rede (em inglês, web-scale discovery systems), as bibliotecas estão oferecendo através de uma única interface, uma única busca e uma única lista de resultados, se não todo, a maioria do conteúdo que possuem em assinaturas, em acesso livre e, inclusive, em seu acervo catalogado. Estas ferramentas oferecem acesso centralizado e mais eficaz ao conteúdo. Esta tendência é também uma resposta à concorrência que as bibliotecas vivenciam com ferramentas de busca como Google e outros sistemas equivalentes que, aliadas ao aumento do uso de dispositivos móveis, propiciam autonomia e agilidade aos pesquisadores. Como observam Kress, Del Bosque e Ipri (2011), os usuários finais esperam que os sistemas das bibliotecas funcionem como as buscas na internet e ficam frustrados quando não respondem da mesma forma, trocando a complexidade dos sites das bibliotecas por buscas no Google.

A Universidade Estadual Paulista Júlio de Mesquita Filho (UNESP), em 2011, foi pioneira no Brasil na implantação do sistema de descoberta Primo produzido pela empresa Ex Libris/ProQuest (VIANA, 2016). No entanto, 
ainda há poucas bibliotecas universitárias utilizando serviços de descoberta no Brasil.

Por um lado, os sistemas de descoberta solucionaram a busca integrada e o acesso a toda coleção da biblioteca. Por outro, os softwares do tipo ILS, para gerenciamento das bibliotecas, não suportam o conteúdo eletrônico e digital, em razão do número crescente de fontes, da variedade e da complexidade de opções de aquisição e licenciamento, das necessidades específicas de registro, de controle e de acesso a estes recursos. Para gerenciamento deste espectro de materiais, no que diz respeito às atividades e competências administrativas, de processos técnicos e de prestação de serviços, as bibliotecas precisam, além dos softwares de bibliotecas do tipo ILS, munir-se de um conjunto de sistemas e ferramentas adicionais, que atendam às novas demandas.

Breeding (2012c) aponta que em razão do modelo tradicional ILS não se prestar para o gerenciamento de recursos eletrônicos, proliferaram produtos complementares para atender este aspecto estratégico das operações das bibliotecas. As bibliotecas universitárias, além dos ILSs, implementam serviços de resolução de links $O p e n U R L$, sistemas para o gerenciamento de recursos eletrônicos, interfaces de descoberta, plataformas para gerenciamento de coleções digitais, repositórios institucionais, servidores de proxy e outros componentes necessários para atender suas demandas. Essa variedade de sistemas "[...] resulta em um ambiente muito complexo de ser gerenciado pelas bibliotecas" (BREEDING, 2012c, p. 11, tradução nossa).

Diante deste cenário, os sistemas de descoberta modernizaram o acesso, mas o gerenciamento de recursos está comprometido por sistemas e práticas antigas. Segundo Levy (2013), o desafio está entre o conteúdo que a biblioteca possui e as formas nas quais este conteúdo está organizado. As bibliotecas necessitam de ferramentas para gerenciamento de suas atividades internas que, em última instância, criam os acessos. Observa-se um ambiente com variedade de recursos e formas de gerenciamento, onde as operações e controles estão distribuídos em diversos sistemas, pois os conteúdos requerem processos diferenciados: material impresso versus eletrônico; recursos locais versus remotos; sistemas instalados versus hospedados; ILSs versus ferramentas para controle de fontes eletrônicas.

Seguindo o desenvolvimento da nova geração de catálogos de bibliotecas na forma de ferramentas de descoberta, bibliotecários e fornecedores perceberam que uma solução definitiva não deveria limitar-se à interface do usuário final da biblioteca. Era também necessário envolver a retaguarda (em inglês, back office ${ }^{5}$ ) ou módulos de trabalho, que, da mesma forma, deveriam evoluir para acompanhar a era digital. (YANG, 2013, p. 3, tradução nossa).

Neste contexto, de crescimento do conteúdo eletrônico e aumento da complexidade para o gerenciamento deste conteúdo, surgiram as

\footnotetext{
${ }^{5}$ Back office são os departamentos administrativos e internos de uma biblioteca, que apoiam as operações necessárias para a prestação de serviços a que a biblioteca se destina.
} 
plataformas de serviços de bibliotecas (GREEN, 2014), que constituem uma nova categoria de sistemas utilizada desde o início desta década, principalmente, em instituições de ensino superior e de pesquisa nos Estados Unidos e na Europa. As características das plataformas de serviços de bibliotecas se diferem substancialmente dos já estabelecidos ILSs, em razão de que estes não cumpriam necessariamente as expectativas, sendo de grande utilidade considerar uma geração totalmente nova de produtos, sem bagagem conceitual dos produtos já existentes:

Uma plataforma de serviços de biblioteca permite às bibliotecas adquirir e gerenciar suas coleções, abrangendo vários formatos de conteúdo, desde itens físicos até materiais eletrônicos. Estes produtos suportam vários processos de aquisição, incluindo a compra de itens permanentes, aqueles com licenças pagas e assinaturas, e aqueles selecionados em fontes de acesso aberto. Oferece um ambiente de gerenciamento de metadados, contemplando vários esquemas conforme apropriado para cada um dos respectivos formatos de materiais, incluindo no mínimo os padrões MARC e Dublin Core. Uma plataforma de serviços de biblioteca pode incluir um serviço de descoberta integrado ou fazer uso de uma interface de descoberta adquirida, apoiando-se em APIs $^{6}$ e outros protocolos de interoperabilidade. As plataformas de serviços são oferecidas através de uma estrutura multi-tenant e provêm informações a toda a equipe e usuários finais via interfaces web. Estes produtos proporcionam acesso a bases de conhecimento que representam o corpo de conteúdo de informação além da coleção específica da biblioteca. (BREEDING, 2015, p. 8, tradução nossa).

Retomando a evolução dos sistemas para gerenciamento de bibliotecas, a partir das quatro gerações descritas por Rowley (2002), pode-se dizer que as plataformas de serviços de bibliotecas não se enquadram nas soluções de "quarta geração", justificando a criação de uma outra categoria, as plataformas de serviços de bibliotecas. Na próxima seção são apresentados os conceitos, as características, os diferenciais e relato sobre os efeitos das soluções de LSP sobre os processos e serviços das bibliotecas.

\section{As plataformas de serviços de bibliotecas}

No artigo "Future of library systems", Grant (2012a) afirma que muitas bibliotecas estão em processo de avaliação da eficiência das ferramentas de automação que utilizam para prestação de serviços dentro e fora de seus limites físicos. Se por um lado, na maioria das bibliotecas, os serviços são mediados por sistemas do tipo ILS para gerenciamento de bibliotecas, por outro, estão as ofertas de uma nova geração de sistemas,

\footnotetext{
${ }^{6}$ API é a sigla de Application Programming Interface ou, em português, Interface de Programação de Aplicativo. Definição extraída de: <https://www.tecmundo.com.br/programacao/1807-o-que-e-api-.htm>. Acesso em: 1 maio 2017. Este trabalho utiliza a sigla API, pois esta é a forma mais frequente.
} 
batizados por Marshal Breeding como "library services platforms (LSP)" (BREEDING, 2016b).

Os primeiros sistemas do tipo plataformas de serviços de bibliotecas surgiram no início desta década no mercado internacional, contemplando o gerenciamento de todos os materiais das coleções - impressos, eletrônicos e digitais. O termo "plataformas de serviços de bibliotecas" aparece sob outras denominações na literatura ao longo do período de 2012 a 2017, como demonstrado no Quadro 1.

Quadro 1 - Denominações dos novos sistemas de gerenciamento de bibliotecas

\begin{tabular}{|c|c|c|c|}
\hline & Trabalho & $\begin{array}{l}\text { Denominação utilizada no } \\
\text { documento original }\end{array}$ & $\begin{array}{l}\text { Denominação em português } \\
\text { (tradução nossa) }\end{array}$ \\
\hline \multirow{6}{*}{ 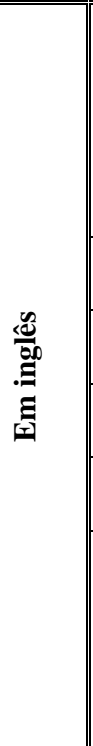 } & $\begin{array}{l}\text { GREEN, } 2014 \\
\text { KEN CHAD CONSULTING, } 2012 \\
\text { LI, } 2014 \\
\text { WANG; DAWES, } 2012 \\
\text { WILSON, } 2012\end{array}$ & next generation library systems & $\begin{array}{l}\text { próxima geração de sistemas de } \\
\text { gerenciamento de bibliotecas }\end{array}$ \\
\hline & $\begin{array}{l}\text { DULA et al., } 2012 \\
\text { OCLC, } 2011 \mathrm{~b}\end{array}$ & web-scale management services & serviços de gerenciamento na web \\
\hline & HOSBURGH, 2014 & uniform management systems & $\begin{array}{l}\text { sistemas de gerenciamento } \\
\text { uniforme }\end{array}$ \\
\hline & KEN CHAD CONSULTING, 2012 & $\begin{array}{l}\text { unified resources management } \\
\text { systems }\end{array}$ & $\begin{array}{l}\text { sistemas de gerenciamento } \\
\text { unificado de recursos }\end{array}$ \\
\hline & LEVY, 2013 & unified management service & $\begin{array}{l}\text { serviços de gerenciamento } \\
\text { unificado }\end{array}$ \\
\hline & $\begin{array}{l}\text { BORDEIANU; KOHL, 2015 } \\
\text { BREEDING, 2011, 2012a, 2013a,2017 } \\
\text { CHAD, 2015 } \\
\text { GRANT, 2012a, 2012b } \\
\text { TYAGI; SENTHIL, } 2015 \\
\text { WILSON, } 2016\end{array}$ & library services platforms (LSP) & $\begin{array}{l}\text { plataformas de serviços de } \\
\text { bibliotecas }\end{array}$ \\
\hline \multirow{2}{*}{ 四 } & VIANA, 2013 & $\begin{array}{l}\text { nova geração de sistemas de } \\
\text { gerenciamento de bibliotecas }\end{array}$ & \\
\hline & $\begin{array}{l}\text { ABADAL; ANGLADA, } 2017 \\
\text { BENTO, } 2013 \\
\text { TONDING; VANZ, } 2016\end{array}$ & $\begin{array}{l}\text { plataformas de serviços de } \\
\text { bibliotecas }\end{array}$ & \\
\hline
\end{tabular}

Fonte: Elaborado pela autora (2017) com base em dados da pesquisa.

No Brasil, o nome do novo sistema não está consolidado: encontram-se as expressões "nova geração de sistemas para bibliotecas", "sistemas de próxima geração", "plataformas de serviços de bibliotecas" e a sigla "LSP". Neste artigo, adota-se a nomenclatura "plataformas de serviços de bibliotecas" e a correspondente sigla LSP - do inglês, library services platform. A escolha se dá por três motivos. Primeiramente pelo fato de que os adjetivos "novo(a)" e "próximo(a)", usados em algumas denominações, tendem a se degradar no tempo e perder sua significação em poucos anos. O segundo motivo leva em conta a escolha feita por Marshall Breeding, um dos autores mais importantes neste tema, que batizou os novos sistemas como library services platforms (LSP) (BREEDING, 2016b). Por último, por causa da ocorrência desta nomenclatura nos artigos mais recentes sobre o tema e da observação da mudança na denominação adotada, como no caso da Ken Chad Consulting 
que passou a usar o nome library services platforms (CHAD, 2015) em substituição à unified resources management systems (KEN CHAD CONSULTING, 2012).

Em 2012, Breeding (2012a) preconizou que as plataformas de serviços de bibliotecas eram um dos principais norteadores da indústria de automação de bibliotecas, que deveriam permanecer no mercado nos próximos 10 anos e que estariam em condições de dar início a um ciclo de migrações. Bordeianu e Kohl (2015) corroboram esta posição, afirmando que a indústria está voltada para as novas plataformas e, aos poucos, vem diminuindo o desenvolvimento e suporte aos antigos ILSs e que, devido a isto, as bibliotecas estão cada vez mais pressionadas a migrarem para os novos sistemas.

As novas plataformas se diferem substancialmente dos ILSs e não podem ser enquadradas na mesma categoria. Entre as características que as distinguem estão o gerenciamento unificado de materiais impressos, digitais e eletrônicos, utilização de bases de conhecimentos globais ao invés de bases de dados locais, implementações através do modelo Software-as-a-Service (Saas) em arquiteturas multi-tenant e o provisionamento de um conjunto de APIs que permitem interoperabilidade e extensibilidade do sistema. A biblioteca necessita utilizar uma variedade de softwares além do ILS para gerenciar todos seus recursos e serviços. Portanto, a implementação dessa nova plataforma pode substituir muitos sistemas, incluindo os ILSs, as ferramentas para gerenciamento de recursos eletrônicos (ERMS), os resolvedores de links OpenURL e os sistemas para gerenciamento de coleções digitais.

Os modelos brasileiros para avaliação de softwares de bibliotecas estabelecidos por Côrte e colaboradores (1999; 2002), por Café, Santos e Macedo (2001) e Grossi (2008) são aplicáveis aos sistemas de quarta geração. Eles foram aplicados por muitas bibliotecas brasileiras como referência no processo de seleção dos sistemas de informatização usados hoje. Estes modelos de análise ainda são válidos se aplicados às opções de software do mercado nacional e dos ILSs de forma geral, mas tornam-se incompletos e, em alguns aspectos, defasados quando submetidos às plataformas de serviços de bibliotecas, que possuem características funcionais inovadoras e são desenvolvidas com tecnologias atuais. Com enfoque mais atual, Viana (2014) apresentou um levantamento de critérios para avaliação de plataformas de serviços de bibliotecas subdivididos em gerenciais e financeiros, biblioteconômicos, computacionais e baseados em serviços e processos. No meio internacional, por sua vez, há iniciativas de metodologias de avaliação como da Ken Chad Consulting que mantém um site $^{7}$ para discussão de especificações de softwares para bibliotecas, incluindo sistemas integrados de bibliotecas (ILS), serviços de descoberta, plataformas de serviços de bibliotecas (LSP) e sistemas para gerenciamento de recursos eletrônicos (ERMS), entre outros.

Observa-se no decorrer desta década, principalmente entre bibliotecas universitárias e de instituições de pesquisa dos Estados Unidos e

\footnotetext{
${ }^{7}$ Site disponível no endereço: <https://libtechrfp.wikispaces.com>. Acesso em: 1 maio 2017.
} 
Europa, o crescente fenômeno de migração de sistemas tradicionais de gerenciamento para plataformas de serviços. Em levantamento realizado junto ao Library Technology Guides ${ }^{8}$, constata-se este crescimento, conforme demonstrado na Tabela 1.

Tabela 1 - Número de instituições que migraram para plataformas de serviços de bibliotecas no período de 2010 a 2017 no mundo

\begin{tabular}{lccccccccc}
\hline Plataforma & $\mathbf{2 0 1 0}$ & $\mathbf{2 0 1 1}$ & $\mathbf{2 0 1 2}$ & $\mathbf{2 0 1 3}$ & $\mathbf{2 0 1 4}$ & $\mathbf{2 0 1 5}$ & $\mathbf{2 0 1 6}$ & $\mathbf{2 0 1 7}$ & Total \\
\hline \hline Ex Libris/ProQuest - Alma & 10 & 8 & 74 & 181 & 71 & 242 & 193 & 6 & 785 \\
OCLC - WorldShare Management & 6 & 39 & 55 & 92 & 75 & 60 & 56 & 1 & 384 \\
$\quad$ Services $($ WMS $)$ & & & & & &
\end{tabular}

Fonte: Adaptado de LIBRARY... (2017, s. p. $)^{9}$.

O Library Technology Guides, em consulta realizada em maio de 2017, apontou os dois sistemas - Alma da Ex Libris/ProQuest e WorldShare Management Services (WMS) da Online Computer Library Center (OCLC) - mencionados na tabela 1, como as únicas soluções de LSP desenvolvidas e comercializadas no momento ${ }^{10}$.

Na literatura, no entanto, encontra-se referências a outros sistemas ditos LSPs. Breeding (2016b), em artigo que revisa os softwares de gerenciamento de bibliotecas após cinco anos do lançamento das primeiras plataformas de serviços de bibliotecas, comenta que, após o longo processo de desenvolvimento e amadurecimento destas soluções, alguns destes sistemas mencionados como LSPs, de fato, não aderem totalmente aos requisitos tecnológicos que caracterizam o gênero. Os produtos Intota da ProQuest e OpenSkies da VTLS, que aparecem em alguns trabalhos, foram descontinuados. Há também referências ao Sierra da Innovative Interfaces Inc. e ao Kuali OLE (solução open source, em português, de código aberto) - porém, hoje os dois são enquadrados na categoria de ILS no diretório mencionado. Ambos foram concebidos com algumas características consistentes ao modelo das plataformas de serviços de bibliotecas, mas não aderem totalmente aos pressupostos tecnológicos. O Sierra, por exemplo, não oferece todas as funcionalidades em interface web e o Kuali OLE, por sua vez, não foi projetado em arquitetura multi-tenant (BREEDING, 2016b). Por último, há que se mencionar o sistema FOLIO, uma iniciativa colaborativa de construção de um software de código aberto do tipo LSP que está em andamento e conta com a adesão de diversas bibliotecas, profissionais bibliotecários e empresas do ramo de tecnologia da informação.

${ }^{8}$ Library Technology Guides é um diretório, criado e mantido por Marshall Breeding, que provê informações sobre "[...] diferentes tipos de tecnologias e serviços usados por bibliotecas. Cobre as organizações que desenvolvem e suportam sistemas e softwares orientados para bibliotecas. Oferece uma base de dados completa e um repositório de documentos para auxiliar as bibliotecas que estão analisando novos sistemas [...]" (LIBRARY..., 2017, s. p., tradução nossa).

9 Informações extraídas do site do Library Technology Guides. Disponível em: <https://librarytechnology.org/products/migration.pl>. Acesso em: 6 maio 2017.

10 Breeding (2017) relata que as tecnologias de bibliotecas estão passando por processos de consolidação vertical (com empresas de produtos complementares) e horizontal (com empresas de produtos concorrentes). Este fenômeno explica o reduzido número de sistemas da categoria LSP atualmente disponíveis. 
No Brasil, não há registro de empresas de softwares de bibliotecas que desenvolvam soluções do tipo plataformas de serviços de bibliotecas. Bem como não há nenhuma biblioteca utilizando sistemas desta categoria. A primeira iniciativa comercial relacionada às plataformas de serviços no país foi o evento Alma Solution Day, realizado em São Paulo, em novembro de 2015, direcionado para bibliotecas universitárias, e que contou com apresentações de gerentes da Ex Libris/ProQuest (Israel, Estados Unidos e Brasil) e de Carl Grant (Associate Dean, Knowledge Services e Chief Technology Officer), que apresentou sua experiência de implementação da plataforma Alma na biblioteca da University of Oklahoma.

Entre as plataformas de serviços de bibliotecas há uma série de semelhanças, mas cada uma delas tem ou teve sua própria visão estratégica, arquitetura técnica, nível de completude e maturidade de funcionalidades. A seguir estão apresentados os pressupostos tecnológicos que caracterizam estes sistemas: abordagem de desenvolvimento; computação em nuvem; Software-as-a-Service em plataformas multitenant; unificação de fluxos de trabalhos; compartilhamento; interoperabilidade e extensibilidade; ferramentas de análise; serviço de descoberta; e, por fim, assinatura e valores.

Abordagem de desenvolvimento é o pressuposto que situa a forma de desenvolvimento do produto: nova concepção, evolução ou código aberto, segundo Grant (2012a). Desde o surgimento das primeiras LSPs, muitos foram os movimentos da indústria de software para gerenciamento de bibliotecas em busca do desenvolvimento de soluções mais contemporâneas. Vê-se a oferta de novos produtos, apoiados em tecnologias mais recentes, com novas características em relação aos clássicos ILSs. No entanto, somente, aqueles sistemas desenvolvidos com o que Grant (2012a) chamou de "nova concepção" - desenvolvidos "a partir do zero", de modo a acomodar necessidades existentes, como por exemplo, integrar perfeitamente os fluxos de trabalho independentemente do tipo de material ser impresso, eletrônico ou digital - é que são considerados compatíveis com a categoria de plataformas de serviços de bibliotecas (BREEDING, 2016b).

Outro pressuposto para as plataformas de serviços de bibliotecas é a computação em nuvem. Mavodza (2013) afirma que, com o crescimento exponencial das fontes de informação e de todas as complexidades que acompanham este processo, a capacidade de hospedagem das bibliotecas em suas próprias estruturas não é mais suficiente. Isto leva a adoção de alternativas na nuvem, processo que faz com que as preocupações sobre hardware e software sejam substituídas por preocupações referentes ao acesso à informação. Apesar das vantagens como sustentabilidade, disponibilidade e praticidade dos sistemas em nuvem, a autora chama atenção para áreas preocupantes como a segurança de dados e usabilidade - questões que devem ser cuidadosamente previstas nos contratos de serviços. A flexibilidade e escalabilidade da computação em nuvem são outras vantagens do modelo destacada por Corrado e Moulaison (2012). Em bibliotecas, a frequência 
de uso dos serviços e recursos varia de acordo com horários e fases, demandando mais ou menos recursos do sistema de informações, dependendo do momento. A nuvem, ao contrário dos servidores locais que precisam ser estimados para os picos de uso e que se tornam subutilizados, oferece, através da tecnologia, flexibilidade para estas variações. Quanto ao aspecto da escalabilidade, os autores dão o exemplo da decisão de implementação de novos serviços na nuvem, pois podem rapidamente dispor de recursos adicionais de computação caso se tornem populares - agilidade que não ocorreria em caso de necessidade de aquisição de novos servidores locais.

As plataformas de serviços de bibliotecas são fornecidas como Software-as-a-Service (SaaS) em plataformas multi-tenant, modelo de utilização de sistemas no qual o software é hospedado pelo fornecedor e o acesso do usuário é por meio da internet. O consumidor, nesse caso, a biblioteca, paga para utilizar o serviço por um determinado tempo ou demanda. "Ao usar SaaS, você está usando uma máquina hospedada remotamente ao invés de uma máquina local e a empresa que hospeda a máquina tem a responsabilidade de manter o sistema [...]" (GRANT, 2012a, p. 5, tradução nossa). Tyagi e Senthil (2015) registram que, neste modelo, os serviços de bibliotecas contratados são acessados via internet no regime $24 \times 7$, ou seja, sem interrupções. Os autores salientam que os fornecedores oferecem serviços hospedados próprios ou contratados de terceiros e enfatizam que, neste segundo caso, os contratos são únicos e englobam a "contratação do servidor para hospedagem e do serviço associado" (TYAGI; SENTHIL, 2015, p. 409, tradução nossa). Uma aplicação multi-tenant atende a diversos clientes a partir de uma única instalação, de modo que configurações, manutenções, atualizações e outras atividades sejam feitas apenas uma vez e aplicadas a todos usuários. Green (2014), no artigo em que relata a experiência de implementação da plataforma de serviços na Curtin University Library, constata que a qualidade SaaS/multi-tenant estimula o crescimento da comunidade de usuários de um serviço e exemplifica isto comentando que, se uma atualização de versão traz algum defeito, todos os clientes sofrerão o impacto, demandando esforço ágil, concentrado e único do fornecedor para resolução do problema. Outro aspecto que o seu artigo salienta é sobre a manutenção ser responsabilidade do fornecedor e não da instituição. Neste sentido, o autor comenta que as atualizações do sistema ocorrem com frequência mensal e sem nenhum envolvimento da equipe, gerando uma transformação no foco da biblioteca que, ao invés de preocupar-se em instalar e testar as novas versões, passou a conhecer e tirar vantagens das funcionalidades implementadas pelas atualizações.

O pressuposto unificação de fluxos de trabalhos refere-se à variedade de acervos e sistemas, que geram duplicação de dados e de trabalho, assim como uma diversidade de caminhos e de interfaces para gerenciamento, busca e acesso a estes recursos. Nas LSPs, a tradicional divisão entre registro de catalogação e registro de coleção física, própria dos ILSs, passa a ser denominada como gerenciamento de metadados e gerenciamento de inventário. O processo de aquisição de materiais 
impressos e eletrônicos é gerenciado usando a mesma plataforma, adaptando os fluxos às exigências de um ou outro material - livros são recebidos, fontes eletrônicas são ativadas; itens são cadastrados, fontes eletrônicas têm suas licenças de uso registradas. No que diz respeito à catalogação dos recursos de informação, as plataformas de serviços são compatíveis com RDA (Resource Description and Access) e os formatos de registro podem ser usados de forma híbrida dependendo do recurso. As LSPs suportam, ao menos, os formatos MARC e Dublin Core, e algumas ampliam para outros formatos, como Encoded Archival Description (EAD), Metadata Object Description Schema (MODS), Metadata Encoding and Transmission Standard (METS), ONIX ${ }^{11}$ entre outros (BREEDING, 2012c, 2013a; WILSON, 2012; YANG, 2013).

O conceito de compartilhamento está fortemente implantado nas plataformas de serviços de bibliotecas, visto que as LSPs abandonam o método de cadastramento de "registro-por-registro", evitando o retrabalho e buscando simplificação e otimização, tanto nos processos de aquisição de materiais, quanto nos processos de gerenciamento de metadados. Breeding (2012c, 2013b) comenta que as novas plataformas incorporam modelos de metadados altamente compartilhados em bases de conhecimento mantidas pelos fornecedores de LSP e outros componentes que podem ser utilizados por todas as bibliotecas usuárias para evitar a replicação de dados (BREEDING, 2016b).

Outro pressuposto importante diz respeito a interoperabilidade e extensibilidade. Neste sentido, as plataformas de serviços de bibliotecas suportam APIs (Application Programming Interface ou, em português, Interface de Programação de Aplicativo) que permitem o desenvolvimento de extensões de modo a atender necessidades específicas da biblioteca e da instituição usuária. Breeding (2015) exemplifica estas extensões com duas situações: as bibliotecas consomem dados gerenciados por outros sistemas, como por exemplo, cadastros de usuários finais provenientes dos sistemas de registro acadêmico e funcional das universidades; as bibliotecas podem alimentar os sistemas financeiros da universidade com dados e transações gerenciadas em seus processos de aquisição. Outro exemplo de utilização de APIs que o autor cita é no caso de extração de dados para uso em uma aplicação externa, com o objetivo de obter maior capacidade de relatórios, incluindo, por exemplo, cálculos estatísticos, análise de dados ou controle de formatação.

Entre as forças que membros da OCLC e especialistas em tecnologia da informação elencaram como formadoras do futuro das bibliotecas em todo o mundo figura "A necessidade de novos tipos de análises e métricas mais vinculadas às medidas de desempenho" (OCLC, 2011a, p. 21, tradução nossa). Grant (2012a) diz que, munidas de ferramentas de análises ou analytics, as bibliotecas podem compreender detalhadamente seus usuários finais e prever, com maior grau de precisão, os tipos de serviços e conteúdos que eles precisam e, inclusive,

${ }^{11}$ ONIX é um formato utilizado no comércio eletrônico para descrição de livros, de periódicos e de termos de licenciamento. Definição extraída de: <http://www.editeur.org/8/ONIX/>. Acesso em: 16 jul. 2017. 
quando irão precisar. Embora os sistemas de gerenciamento de bibliotecas normalmente incluam um conjunto de relatórios, as plataformas de serviços contemplam capacidades mais avançadas de análise e avaliação de coleções. O fato das LSPs unificarem o processamento de todo o acervo da biblioteca contribui para manipulação dos dados com uma visão integrada de toda a coleção.

As plataformas de serviços de bibliotecas também se integram aos serviços de descoberta. Segundo Breeding (2015) os serviços de descoberta e as plataformas de serviços de bibliotecas são duas categorias de produtos, mas os dois não podem operar de forma independente. Os conteúdos e as funcionalidades que residem na plataforma de serviços da biblioteca são apresentados aos usuários finais através da interface de descoberta.

Comercializadas através do modelo de negócio de assinatura, as plataformas de serviços de bibliotecas diferem-se dos ILSs com instalação local, pois estes aplicam um valor inicial de licenciamento e uma taxa de manutenção e atualização anual. Conforme Breeding (2015), as implementações do tipo SaaS são normalmente oferecidas através de assinaturas anuais cujos valores dependem do tamanho e da complexidade da instituição. Nos valores das assinaturas estão embutidos custos de hardware, de sistemas operacionais, de infraestrutura de data center e de pessoal técnico, sendo, em geral, superiores às taxas de manutenção associadas com os sistemas instalados localmente. No entanto, segundo o autor, o custo total das implementações de LSP, quando todas as despesas e investimentos são calculados, equiparam-se às cobradas pelos ILSs.

Para concluir, apresenta-se de forma resumida os atributos que, segundo Breeding (2016b), qualificam as plataformas de serviços de bibliotecas:

a) são implementados como SaaS (sem instalação de servidor local ou software);

b) usam arquitetura multi-tenant, ou seja, todos os usuários do serviço compartilham uma mesma instância do software (uma única instalação, com a mesma versão para todos);

c) todas as funcionalidades são acessadas através de um navegador da internet;

d) gerenciam materiais impressos, eletrônicos e digitais com uma abordagem unificada, criando fluxos diferenciados quando necessário;

e) possuem uma base de conhecimento própria para recursos eletrônicos, bem como um catálogo comum de registros bibliográficos;

f) oferecem APIs para interoperabilidade e extensibilidade.

\section{Considerações finais}


A implementação de qualquer produto de automação de bibliotecas é um processo complexo. Estes sistemas interferem em diversas atividades da biblioteca e, por isto, uma mudança requer cautela para que não haja transtornos na transição e demanda que a biblioteca tenha confiança sobre os benefícios do novo sistema. A decisão de adoção de um software e, mais ainda, de um sistema recente e contemporâneo, requer uma fundamentação sobre as necessidades de implantação e sobre os benefícios que a mudança trará. A opção também exige argumentos e indicadores para justificar, junto aos gestores, os investimentos necessários.

No cenário das bibliotecas universitárias brasileiras e no que diz respeito aos movimentos tecnológicos que ocorrem neste meio, observa-se, com mais frequência, o interesse das bibliotecas em modernizarem os sistemas voltados aos usuários finais, com a adoção dos sistemas de descoberta em substituição aos tradicionais OPACs. Vale destacar, porém, que a literatura demonstra que o uso de ferramentas fragmentadas para gerenciamento das coleções, combinadas com soluções de descoberta para recuperação e acesso à informação são típicos de uma fase anterior à adoção dos sistemas LSPs.

As plataformas de serviços de bibliotecas, desenvolvidas com tecnologias recentes, são providas através de serviços em nuvem e com funcionalidades mais completas e modernas para o gerenciamento unificado dos mais diversos recursos de informação hoje existentes nas bibliotecas. Por este motivo, considera-se que apresentação de uma pesquisa bibliográfica sobre as LSPs traz benefícios para a área da biblioteconomia, tendo em vista que consiste em uma temática nova internacionalmente e sobre a qual há escassez de literatura científica, especialmente em português. Espera-se gerar conhecimentos sobre o tema que contribuam na tomada de futuras decisões sobre a avaliação das plataformas de serviços de bibliotecas.

A realização de uma pesquisa empírica de caráter qualitativo é a próxima etapa do estudo. Aprofundar a análise, mergulhar no universo pesquisado e conhecer o fenômeno em profundidade, certamente, trará contribuições com maior valor para um tema ainda pouco estudado e, ao mesmo tempo, tão proeminente.

\section{Referências}

ABADAL, E.; ANGLADA, L. TIC e bibliotecas: situação atual e perspectivas. In: LEMOS, Anna Carolina Mendonça; FERREIRA, Pedro Cavalcanti Gonçalves Ferreira (Org.). Biblioteca do século XXI: desafios e perspectivas. Brasília, DF: IPEA, 2017. p. 301-326. Disponível em: <http://www.ipea.gov.br/agencia/images/stories/PDFs/livros/livros/17010 5_biblioteca_do_seculo_21.pdf >. Acesso em: 1 maio 2017.

ANDERSON, E. K. Elements of electronic resource management. Library Technology Reports, Chicago, v. 50, n. 2, p. 11-22, Apr. 2014. Disponível 
em: <https://journals.ala.org/index.php/ltr/article/view/4492/5256>. Acesso em: 30 abr. 2017.

APPS, A..; MACINTYRE, R.. Why OpenURL? 25 Nov. 2005. s.p. Paper para acompanhar a apresentação de Ross MacIntyre no STM Innovations Seminar, Londres, 2005. Disponível em: <http://epub.mimas.ac.uk/papers/stm2005/appsmac-whyopenurl200511_full.html>. Acesso em: 21 maio 2017.

BENTO, F. Para onde evoluem os sistemas de gestão integrada de bibliotecas: dos formatos à nuvem. Aveiro: [s.n.], 2013. 29 p. Trabalho apresentado no 2. Encontro de Bibliotecas do Ensino Superior, Aveiro, 2013. Disponível em: <https://www.bad.pt/2encontrobes/wpcontent/uploads/2013/06/WS_Sistemas_FilipeBento.pdf $>$. Acesso em: 15 jul. 2017.

BORDEIANU, S; KOHL, L. The Voyage Home: New Mexico libraries migrate to WMS, OCLC's cloud-based ILS. Technical Services Quarterly, NewYork, v. 32, n. 3, p. 274-293, Jun. 2015.

BREEDING, M. A cloudy forecast for libraries. Computers in Libraries, Westport, v. 31, n. 7, p. 32-34, Sept. 2011.

BREEDING, M. Agents of change: automation product vendors are poised for a major transition. Library Journal, New York, NY, v. 137, n. 6, p. 30-36, Apr. 2012a.

BREEDING, M. E-resource knowledge bases and link resolvers: an assessment of the current products and emerging trends. Insights: the UKSG journal, [Reino Unido], v. 25, n. 2, p. 173-182, Jul. 2012b.

BREEDING, M. Current and future trends in information technologies for information units. El Profesional de la Información, Barcelona, v. 21, n. 1, p. 9-15, enero/feb. 2012c.

BREEDING, $M$. Beyond the ILS: a new generation of library services platforms. In: IGLESIAS, E. (Ed.). Robots in academic libraries: advancements in library automation. Hershey, PA: Information Science Reference, 2013a. p. 13-36. Disponível em: <https://librarytechnology.org/docs/17802.pdf>. Acesso em: 24 out. 2016.

BREEDING, M. The evolving role of the librarian: trends and issues in technology and collection management. 15 Feb. 2013b. 47 slides. Apresentação em Microsoft PowerPoint. Disponível em: <http://librarytechnology.org/docs/17834.pptx>. Acesso em: 17 jul. 2017.

BREEDING, M. Library services platform: a mature genre of products. Library Technology Reports, Chicago, v. 51, n. 4, May/Jun. 2015. 40 p.

BREEDING, M. Smarter libraries through technology: five years of library services platforms. Smart Libraries, Chicago, v. 36, n. 8, p. 1-7, Aug. 2016b. 
BREEDING, M. Library Systems Report 2017: competing visions for technology openness, and workflow. American Libraries, Chicago, v. 48, n. 5, p. 22-35, May 2017. A versão completa dos dados estatísticos apresentados no artigo está disponível em: $<$ https://americanlibrariesmagazine.org/wp-

content/uploads/2017/04/library-sytems-charts.pdf>. Acesso em: 7 maio 2017.

CAFE, L.; SANTOS, C. dos; MACEDO, F. Proposta de um método para escolha de software de automação de bibliotecas. Ciência da Informação, Brasília, DF, v. 30, n. 2, p. 70-79, maio/ago. 2001.

CHAD, K. Library management system to library services platform: resource management for libraries: a new perspective. [S.I.] : Ken Chad Consulting, 2015. 12 p. (Higher Education Library Technology Briefing paper, 1). Disponível em: <http://helibtech.com/file/view/Resource_management_briefing_HELibTe ch_KenChad_Aug2015.pdf/560007613/Resource_management_briefing_H ELibTech_KenChad_Aug2015.pdf>. Acesso em: 23 fev. 2016.

CHAD, K. Rethinking the library services platform. [S.I.] : Ken Chad Consulting, 2016. 10 p. (Higher Education Library Technology Briefing paper, 2). Disponível em: <http://helibtech.com/file/view/Rethinking_the_LSP_Jan2016a.pdf/57190 3939/Rethinking_the_LSP_Jan2016a.pdf>. Acesso em: 21 maio 2017.

CHIEN, S.-W.; TSAUR, S.-M. Investigating the success of ERP systems: case studies in three Taiwanese high-tech industries. Computers in Industry, Amsterdam, v. 58, n. 8/9, p. 783-793, Dec. 2007.

CORRADO, E. M.; MOULAISON, H. L. The library cloud pros and cons. The Digital Shift, 5 Mar. 2012. s. p. Disponível em: <http://www.thedigitalshift.com/2012/03/software/the-library-cloudpros-and-cons/>. Acesso em: 14 abr. 2017.

CÔRTE, A. R. e et al. Automação de bibliotecas e centros de documentação: o processo de avaliação e seleção de softwares. Ciência da Informação, Brasília, DF, v. 28, n. 3, p. 241-256, set./dez. 1999.

CÔRTE, A. R. e et al. Avaliação de softwares para bibliotecas e arquivos: uma visão do cenário nacional. 2. ed. rev. e ampl. São Paulo: Polis, 2002. $221 \mathrm{p}$.

DULA, $M$. et al. Implementing a new cloud computing library management service: a symbiotic approach. Computers in Libraries, Westport, v. 32, n. 1, p. 6-40, Jan./Feb. 2012.

GOLDNER, Matt. Winds of change: libraries and cloud computing. BCLA Browser: linking the library landscape, v. 4, n. 1, 2012. s. p. Disponível em: <http://bclabrowser.ca/index.php/browser/article/view/362/464>. Acesso em: 24 out. 2016. 
GRANT, C. The future of library systems: library services platforms. Information Standards Quarterly, v. 24, n. 4, p. 4-15, Fall 2012a. Disponível em: <http://www.niso.org/sites/default/files/stories/201709/FE_Grant_Future_Library_Systems_\%20isqv24no4.pdf>. Acesso em: 23 abr. 2017.

GRANT, C. Impressions of the new library service platforms: part 1. Thoughts from Carl Grant, 22 Out. 2012b. s.p. Disponível em: <http://thoughts.care-affiliates.com/2012/10/impressions-of-new-libraryservice.html>. Acesso em: 31 ago. 2015.

GREEN, P. Implementing a next generation library system. In: INTERNATIONAL ASSOCIATION OF SCIENTIFIC AND TECHNOLOGICAL UNIVERSITY LIBRARIES CONFERENCE, 35., 2014, Espoo. Proceedings... Espoo: Purdue University, 2014. 7 p. (Purdue e-pubs). Disponível em: $<$ http://docs. lib.purdue.edu/cgi/viewcontent.cgi ?article=2040\&context=ia tul>. Acesso em: 23 fev. 2016.

GROSSI, M. G. R. Estudo das características de software e implementação de um software livre para o sistema de gerenciamento de bibliotecas universitárias federais brasileiras. 2008. 253 f. Tese (Doutorado em Ciência da Informação) - Escola de Ciência da Informação, Universidade Federal de Minas Gerais, Belo Horizonte, 2008. Disponível em: <http://hdl.handle.net/1843/EARM-7H8M9J>. Acesso em: 15 jun. 2017.

HOSBURGH, N. Unified resource management. [Winter Park: Rollins College], 2014. 7 p. Trabalho apresentado no Independent Colleges \& Universities of Florida (ICUF) Meeting, 2014. Disponível em: $<$ https://www.academia.edu/7174717/Unified_Resource_Management_Syste ms?auto=download $>$. Acesso em: 23 fev. 2016.

KEN CHAD CONSULTING. Specification for a unified (next generation) library resource management system. Versão 2. London, 2012. 21 p. Disponível em:

<http://libtechrfp.wikispaces.com/file/view/Unified_library_resource_man agement_system_specification_v2_Aug2012.doc/356577770/Unified_libra ry_resource_management_system_specification_v2_Aug2012.doc>.

Acesso em: 23 fev. 2016.

KRESS, N., DEL BOSQUE, D.; IPRI, T. User failure to find known library item. New Library World, London, v. 112, n. 3/4, p. 150-170, 2011.

LEVY, R. Library in the cloud with diamonds: a critical evaluation of the future of library management systems. Library Hi Tech News, Ann Arbor, v. 30, n. 3, p. 9-13, 2013.

LI, X. What would be the future of the integrated library systems? In: INTERNATIONAL ASSOCIATION OF SCIENTIFIC AND TECHNOLOGICAL UNIVERSITY LIBRARIES CONFERENCE, 35., 2014, Espoo. Proceedings... Espoo: Purdue University. 2014. 9 p. (Purdue e-pubs). Disponível em: $<$ http://docs. lib.purdue.edu/cgi/viewcontent.cgi?article=2042\&context=ia tul/>. Acesso em: 17 nov. 2016. 
LIBRARY TECHNOLOGY GUIDES: documents, databases, news, and commentary. [S.I.]: Marshall Breeding, 2017. s. p. Disponível em: <https://librarytechnology.org>. Acesso em: 6 maio 2017. Site atualizado frequentemente, sem uma data de publicação fixa.

MAVODZA, J. The impact of cloud computing on the future of academic library practices and services. New Library World, London, v. 114, n. 3/4, p. 132-141, 2013.

NOE, D. Open Source Electronic Resource Management Systems (ERMs). Santa Barbara: ByWater Solutions, 2013. s. p. Disponível em: <http://bywatersolutions.com/2013/06/07/open-source-electronicresource-management-erms/>. Acesso em: 30 abr. 2017.

OCLC. Libraries at webscale: a discussion document. Dublin, 2011a. 67 p. Disponível <http://www.oclc.org/content/dam/oclc/reports/worldsharemanagement-services/libraries-at-webscale.pdf>. Acesso em: 23 fev. 2016.

OCLC. 32 libraries lead the way to web-scale innovation: OCLC Web-scale Management Services on target for July 1 general release. Dublin, 2011b. 66 p. Disponível

em: <https://www.oclc.org/de/news/releases/2011/201128.html>. Acesso em: 25 abr. 2017.

PAVÃO, C. M. G. Comportamento de busca e recuperação da informação em serviços de descoberta em rede no contexto acadêmico. 2014. 225 f. Tese (Doutorado em Comunicação e Informação)- Faculdade de Biblioteconomia e Comunicação, Universidade Federal do Rio Grande do Sul, Porto Alegre, 2014. Disponível em: <http://hdl.handle.net/10183/96705>. Acesso em: 3 abr. 2016.

ROWLEY, J. A biblioteca eletrônica. Brasília, DF: Briquet de Lemos/Livros, 2002. 399 p.

SAYÃO, L. F. Interoperabilidade das bibliotecas digitais: o papel dos sistemas de identificadores persistentes - URN, PURL, DOI, Handle System, CrossRef e OpenURL. Transinformação, Campinas, v. 19, n. 1, p. 65-82, abr. 2007.

TONDING, F. J.; VANZ, S. A. de S. As bibliotecas universitárias brasileiras nas nuvens: plataformas de serviços para gerenciamento de bibliotecas. In: SEMINÁRIO NACIONAL DE BIBLIOTECAS UNIVERSITÁRIAS, 19., 2016, Manaus. Anais... Manaus: UFAM, 2016. 12 p. Disponível em: <http://periodicos.ufam.edu.br/anaissnbu/article/view/3186/2829>. Acesso em: 6 jan. 2017.

TYAGI, A. K.; SENTHIL, V. Library automation in India: assessment of library services platforms. DESIDOC Journal of Library \& Information Technology, Delhi, v. 35, n. 6, p. 408-416, Nov. 2015. 
VAN DE SOMPEL, H.; BEIT-ARIE, O. Open linking in the scholarly information environment using the OpenURL framework. New Review of Information Networking, Oxfordshire, v. 7, n. 1, p. 59-76, 2001.

VIANA, M. M. M. Nova geração de sistemas para uma nova geração de bibliotecas. 2013.250 slides. Disponível em: <http://pt.slideshare.net/miquemv/nova-geracaodesistemasparabibliotecas2013viana>. Acesso em: 4 abr. 2015.

VIANA, Michelângelo Mazzardo Marques. Sistemas de informação para bibliotecas universitárias: os requisitos dos gestores, dos bibliotecários e da equipe de tecnologia. Belo Horizonte, 2014. 63 slides. Minicurso apresentado no 18. Seminário Nacional de Bibliotecas Universitárias, Belo Horizonte, $2014 . \quad$ Disponível em: <http://pt.slideshare.net/miquemv/sistemas-de-informao-para-bibliotecasuniversitrias-anlise-multicritrios-2014>. Acesso em: 8 abr. 2015.

VIANA, M. M. M. Uma breve história da automação de bibliotecas universitárias no Brasil e algumas perspectivas futuras. Revista IberoAmericana de Ciência da Informação, Brasília, DF, v. 9, n. 1, p. 4386, jan./jun. 2016.

WANG, Y.; DAWES, T. A. The next generation integrated library system: a promise fulfilled. Information Technology and Libraries, v. 31, n. 3, p. 76, Sept. 2012.

WEITZEL, S. da R. O papel dos repositórios institucionais e temáticos na estrutura da produção científica. Em Questão, Porto Alegre, v. 12, n. 1, p. 51-71, jan./jun. 2006.

WILSON, $\mathrm{K}$. Introducing the next generation of library management systems. Serials Review, Greenwich, v. 38, n. 2, p. 110-123, Jun. 2012.

WILSON, K. Knowledge bases and library services platforms. Library Technology Reports, Chicago, v. 52, n. 6, p. 13-17, Aug./Sept. 2016.

YANG, S. From integrated library systems to library management services: time for change? Library Hi Tech News, Ann Arbor, v. 30, n. 2, p. 1-8, Mar. 2013. 